\title{
Cardiovascular Outcomes in African Americans with Sickle Cell Trait and Chronic Kidney Disease
}

\author{
Kabir O. Olaniran ${ }^{a}$ Nwamaka D. Eneanya ${ }^{b}$ Andrew S. Allegretti ${ }^{a}$ \\ Sophia H. Zhao ${ }^{a}$ Maureen M. Achebe ${ }^{c}$ Ravi I. Thadhani ${ }^{a, d}$ \\ a Division of Nephrology, Massachusetts General Hospital, Harvard Medical School, Boston, MA, USA; \\ ${ }^{b}$ Renal-Electrolyte and Hypertension Division, Perelman School of Medicine, University of Pennsylvania, \\ Philadelphia, PA, USA; 'CHematology Division, Brigham and Women's Hospital, Harvard Medical School, \\ Boston, MA, USA; ${ }^{d}$ Department of Biomedical Sciences, Cedars-Sinai Medical Center, Los Angeles, CA, USA
}

\section{Keywords \\ Sickle cell trait · Chronic kidney disease · Cardiovascular disease · African Americans · Sex}

\begin{abstract}
Background: Sickle cell trait (SCT) is common among African Americans and has been historically considered to be benign. Recently, SCT has been associated with an increased risk for chronic kidney disease (CKD) and cardiovascular disease in the general population. Our understanding of SCT has been extrapolated largely from data of patients with sickle cell disease (SCD). Notably, in SCD, the outcomes differ by sex. The effect of SCT on cardiovascular risk in the African American CKD population is unknown, and the interaction between $\mathrm{SCT}$ and sex on cardiovascular risk has not been investigated. Methods: We performed a 2-center retrospective cohort study of all African American patients with SCT using international classification of disease diagnosis codes and CKD (using the 2012 Kidney Disease Improving Global Outcomes criteria) with at least 1 year of follow-up between January 2005 and December 2017. A reference group of African American CKD patients without SCT was used as a
\end{abstract}

comparator during the same period. SCT patients and the reference patients were matched at baseline for age, sex, comorbidities, and proteinuria. Primary outcomes were incident coronary artery disease (CAD), incident stroke, and allcause mortality. Analysis of effect modification between sex and SCT on primary outcomes was performed. Results: We identified 621 African American CKD patients, 217 SCT patients, and 404 reference patients. The mean age was $56 \pm 13$ years and $66 \%$ were female. The mean estimated glomerular filtration rate was $69 \pm 30 \mathrm{~mL} / \mathrm{min}$. The mean follow-up time was $8 \pm 4$ years. There were no significant differences in the primary outcomes comparing SCT patients to matched controls. The interaction term between SCT and sex, however, was significant in the CAD model $(p<0.01)$. Stratification by sex showed no increased risk in females but a significantly increased risk for CAD in male SCT patients (hazard ratio [HR] 2.14; $95 \% \mathrm{Cl} 1.18-3.86)$, which persisted after multivariable analysis (HR 2.13; 95\% Cl 1.17-3.86). Conclusion: $\mathrm{SCT}$ is associated with an increased risk for CAD in African American males with CKD. The excess risk in males with SCT appears to follow the same pattern as risk in males with SCD. Larger studies are needed to confirm these findings.

() 2019 S. Karger AG, Basel

\section{KARGER}

(C) 2019 S. Karger AG, Basel 


\section{Introduction}

Sickle cell trait (SCT) is characterized by $20-45 \%$ abnormal hemoglobin $\mathrm{S}$ on hemoglobin electrophoresis and is prevalent among persons of African ancestry. SCT affects 1 in 13 African American births or approximately 3.8 million African Americans in the United States $[1,2]$. Historically, this condition has been thought to be benign as life expectancy is similar to the general population [3]. However, SCT patients have elevated levels of systemic inflammatory biomarkers that suggest a tendency towards endothelial dysfunction, cardiovascular and thromboembolic risk $[4,5]$.

The American Heart Association has listed SCT as a non-traditional risk factor for sudden cardiac death and has advocated for longitudinal studies to establish the overall role and mechanism of SCT in cardiovascular disease (CVD) [6]. Furthermore, recent studies have found SCT to be an independent risk factor for the development of incident chronic kidney disease (CKD), albuminuria, and progression to end-stage renal disease [7-9]. CKD is a well-defined risk factor for cardiovascular events [10], but the characterization of a potential additional cardiovascular risk in the population with SCT and CKD has not been defined. Notably, in sickle cell disease (SCD), which is characterized by $80-95 \%$ abnormal hemoglobin $\mathrm{S}$ on hemoglobin electrophoresis, sex plays a significant role in morbidity and mortality [11-14]. Adult males with SCD have significantly shorter survival and higher rates of pain crises compared to females with SCD [11-14].

Given the high prevalence of SCT in the United States and the disproportionate risk for CVD and CKD in African American patients $[15,16]$, characterizing the potential additive risk in this population is essential for early identification and modification of cardiovascular risk factors. Herein we sought to examine the cardiovascular risk in African American CKD patients with SCT compared to African American CKD patients without SCT - with a specific focus on the interaction between SCT and sex on cardiovascular outcomes. We hypothesized that male African Americans with SCT and CKD would be at an increased risk for cardiovascular events, given their increased risk of complications in SCD [11-14].

\section{Materials and Methods}

\section{Study Population}

We conducted this study using the Partners Healthcare Research Patient Data Registry. Research Patient Data Registry is a centralized clinical data registry that gathers detailed inpatient and outpatient clinical and laboratory data from the member hospitals and institutions of Partners Healthcare, Boston, MA, USA. This retrospective data registry has been described in detail in previously published studies [17-19]. Data were obtained from 2-member hospitals of Partners Healthcare (Massachusetts General Hospital and Brigham and Women's Hospital).

We screened for CKD using the International Classification of Disease, tenth edition (ICD-10) diagnosis codes and their ICD-9 equivalents. We then confirmed the diagnosis of CKD via chart review using 2012 Kidney Disease Improving Global Outcomes criteria [20]. Kidney Disease Improving Global Outcomes criteria stage 1 was confirmed only using albuminuria. The estimated glomerular filtration rate (eGFR) was calculated using the CKD-Epidemiology Collaboration creatinine equation $[21,22]$.

We applied the following exclusion criteria: (i) patients who did not self-identify as African American, (ii) patients with only acute kidney injury [23], (iii) patients with ICD codes for SCD (see appendix), (iv) patients with a cardiovascular event prior to the onset of CKD or prior to January 1, 2005 if CKD was present by that date, and (v) less than 12 months of follow-up in the database. End of follow-up was defined as dialysis start date, date of death, date of last available serum creatinine or December 31, 2017. Each SCT patient was matched to 2 reference patients based on the following baseline criteria: (i) age \pm 3 years, (ii) sex, (iii) CKD stage (1/2,3, or $4 / 5$ ), (iv) hypertension, (v) diabetes mellitus, (vi) current smoker, and (vii) urine albumin: creatinine ratio (urine albumin: creatinine ratio $<30 \mathrm{mg} / \mathrm{g}, \geq 30 \mathrm{mg} / \mathrm{g}$ and missing). Patient selection is displayed in Figure 1.

This study was approved by the Institutional Review Board at Partners Healthcare, Boston and the need for informed consent was waived.

\section{Exposure}

We defined our exposure group as African Americans with CKD who have an ICD code for SCT (see appendix). The reference group was defined as African American patients with CKD and no ICD code for SCT.

\section{Outcomes}

We defined our primary outcomes as: (i) incident coronary artery disease (CAD), (ii) incident stroke, and (iii) all-cause mortality. CAD and stroke events were adjudicated using algorithms based on ICD codes (see appendix). Mortality was confirmed by chart review, and review of the Social Security Death Index.

\section{Covariates}

Baseline characteristics were determined at the time of first available serum creatinine measurement between January 1, 2005 and December 31, 2017. Demographics and current smoking status were obtained by chart review. Comorbidities of interest were obtained using algorithms based on ICD codes (see appendix). Laboratory values were obtained by chart review.

\section{Statistical Analysis}

All analyses were conducted using STATA 14 (StataCorp., College Station, TX, USA). We compared categorical variables using chi-square or Fisher's exact test, and continuous variables using Student $t$ test or Wilcoxon rank sum test depending on the distri-
Olaniran/Eneanya/Allegretti/Zhao/ Achebe/Thadhani 


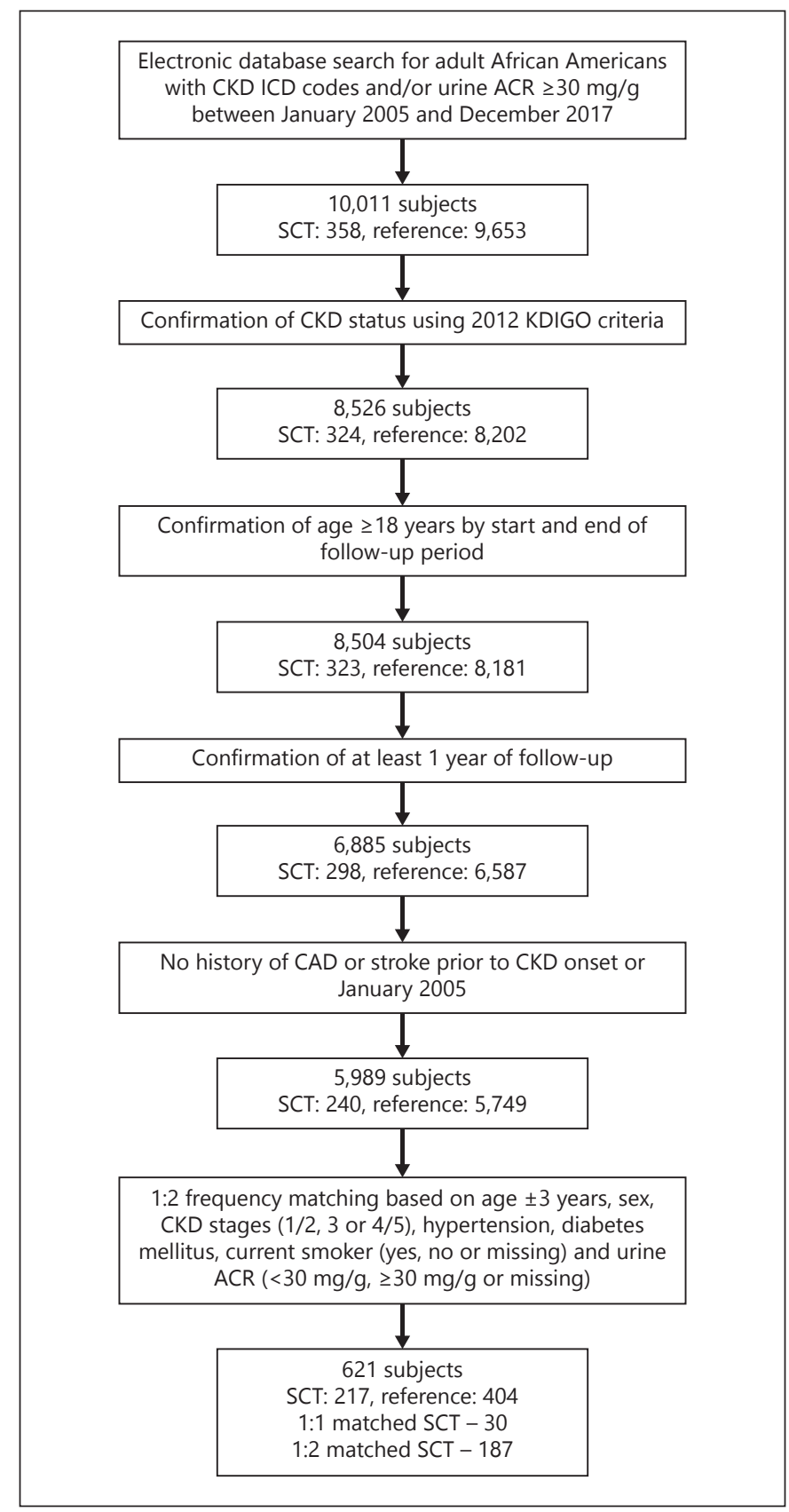

Fig. 1. Flow chart of inclusion in study. CKD, chronic kidney disease; ICD, international classification of disease; ACR, albumin: creatinine ratio; SCT, sickle cell trait; KDIGO, kidney disease improving global outcomes; $\mathrm{CAD}$, coronary artery disease.

bution of data. Censoring was performed at: (i) date of dialysis initiation (due to changing risk profile) or (ii) date of last available serum creatinine. Results of Cox proportional hazard models of the association between SCT and primary outcomes were summarized with hazard ratios (HRs) and Wald asymptotic 95\% CIs. Robust sandwich variance estimates were calculated to account for dependence among matched individuals. Multivariable models

Cardiovascular Outcomes in African

American SCT Kidney Disease were adjusted for age, sex, hypertension, diabetes mellitus, and eGFR where appropriate to account for residual confounding because there were 30 (14\%) SCT patients matched to one reference patient instead of two. Covariates with missing values were excluded from final models. Interactions between SCT and sex, and their effect on each outcome was assessed. We performed analysis stratified by sex for each primary outcome. To address validity of reported ICD codes for SCT, we performed a sensitivity analysis where we examined outcomes only among those with a documented hemoglobin electrophoresis. Two-sided $p$ values $\leq 0.05$ were considered statistically significant.

\section{Results}

\section{Baseline Characteristics}

We identified 621 African American CKD patients of whom 217 had SCT and 404 were in the reference group (Fig. 1). We achieved 1:2 matching in 187 SCT patients with all remaining SCT patients achieving 1:1 matching. Baseline characteristics for all cohort participants are displayed in Table 1 . The mean age of all participants was $56 \pm 13$ years. The mean follow-up for the entire cohort was $8 \pm 4$ years. There were no significant differences in age, sex, body mass index, current smoking status, comorbidities, and laboratory values between patients with SCT versus the reference group. Baseline characteristics for all cohort participants stratified by sex are displayed in Table 2. Overall, there were no significant differences in comorbidities or lab values between female SCT patients and female reference patients. Male SCT patients and male reference patients were also similar.

\section{Association of SCT with Incident CAD, Stroke and Death}

Across all patients, $19 \%(n=121)$ had incident CAD, $14 \%(n=87)$ had incident stroke, and $8 \%(n=50)$ died during follow-up. Using univariate and multivariate Cox regression, the risk of CAD in SCT was not significant (HR 1.13; 95\% CI 0.78-1.64 and HR 1.16; 95\% CI 0.79-1.68, respectively; Table 3). The HR for stroke risk in SCT was $0.73 ; 95 \%$ CI $0.46-1.17$ on univariate analysis and remained unchanged after multivariable analysis. Likewise, the mortality risk in SCT was HR 1.51 (95\% CI 0.87-2.64) on univariate analysis with no change on multivariable analysis.

We evaluated for interactions between SCT and sex in the various adjusted models. In the CAD model, after adjusting for age, sex, hypertension, diabetes mellitus, and eGFR, the interaction term between SCT and sex was significant $(p<0.01)$. In the stroke model, after multivariable adjustment, the interaction term between SCT and 
Table 1. Baseline characteristics of cohort

\begin{tabular}{llll}
\hline Covariate & $\begin{array}{l}\text { SCT } \\
(n=217)\end{array}$ & $\begin{array}{l}\text { Reference } \\
(n=404)\end{array}$ & $p$ value \\
\hline
\end{tabular}

\begin{tabular}{|c|c|c|c|}
\hline \multicolumn{4}{|l|}{ Demographics } \\
\hline $\begin{array}{l}\text { Age, years, } \\
\text { mean (SD) }\end{array}$ & $55(13)$ & $56(13)$ & 0.56 \\
\hline Age $\geq 65$ years, $\%$ & 24 & 26 & 0.63 \\
\hline Female, $\%$ & 67 & 66 & 0.92 \\
\hline \multicolumn{4}{|l|}{ Follow-up, years, } \\
\hline $\mathrm{BMI}, \mathrm{kg} / \mathrm{m}^{2}(\mathrm{SD})^{*}$ & $33(8)$ & $33(9)$ & 0.63 \\
\hline \multicolumn{4}{|l|}{ Comorbidities } \\
\hline Hypertension, \% & 78 & 78 & 0.92 \\
\hline Diabetes mellitus, \% & 58 & 60 & 0.67 \\
\hline CKD, \% & & & 0.91 \\
\hline KDIGO stage 1 & 24 & 24 & \\
\hline KDIGO stage 2 & 20 & 20 & \\
\hline KDIGO stage 3 & 48 & 50 & \\
\hline KDIGO stage 4 & 5 & 4 & \\
\hline KDIGO stage 5 & 3 & 2 & \\
\hline Current smoker, \% & 7 & 6 & 0.91 \\
\hline \multicolumn{4}{|l|}{ Laboratory values } \\
\hline $\begin{array}{l}\text { eGFR, } \mathrm{mL} / \mathrm{min} \text {, } \\
\text { mean }(\mathrm{SD})\end{array}$ & $68(30)$ & $70(29)$ & 0.57 \\
\hline $\mathrm{GFR} \geq 60 \mathrm{~mL} / \mathrm{min}, \%$ & 44 & 44 & 1.00 \\
\hline $\begin{array}{l}\text { Urine } A C R, \mathrm{mg} / \mathrm{g} \\
\text { median (IQR)* }\end{array}$ & $64(144)$ & $59(121)$ & 0.36 \\
\hline Urine ACR $\geq 30$ & & & \\
\hline $\mathrm{mg} / \mathrm{g}, \% *$ & 63 & 62 & 1.00 \\
\hline
\end{tabular}

* Missing data were noted for BMI (152 patients) and urine albumin: creatinine ratio (120 patients).

Percentage values for variables with missing data were calculated among the whole cohort.

BMI, body mass index; CKD, chronic kidney disease; KDIGO, Kidney Disease Improving Global Outcomes; eGFR, estimated glomerular filtration rate; ACR, albumin creatinine ratio; SCT, sickle cell trait.

sex was not significant $(p=0.18)$. Similarly, after multivariable adjustment in the mortality risk model, the interaction term between SCT and sex was not significant $(p=$ $0.79)$.

Once stratified by sex, CAD risk in female SCT patients was not significantly increased compared to female reference patients before (HR 0.76; 95\% CI 0.46-1.26; Fig. 2; Table 4) or after adjustment (HR 0.77; 95\% CI 0.47-1.26; Fig. 3; Table 4). The risk for stroke and mortality in female SCT patients compared to female reference patients remained unchanged (Table 4). Among male SCT patients, the risk for CAD was significantly increased (HR 2.14; 95\% CI 1.18-3.86; Fig 2; Table 4). This association remained significant after adjusting for age, hyper- tension, diabetes mellitus, and eGFR (HR 2.14; 95\% CI 1.18-3.90; Fig. 3; Table 4). There was no association between SCT status and stroke or mortality before or after adjustment in the male sub-cohort (Fig. 2, 3; Table 4).

A total of 61 patients had a documented hemoglobin electrophoresis. All 61 patients had appropriate ICD codes for their exposure status. Sensitivity analysis of this sub-cohort of 61 patients with a documented hemoglobin electrophoresis to verify the presence of SCT or the presence of normal hemoglobin profile (reference group) was mostly similar in terms of direction of effect estimate (appendix Table A1). The main difference was that the overall CAD risk point estimate (HR 0.88; 95\% CI 0.32-2.37) was in a different direction although CAD risk point estimates stratified by sex were essentially unchanged (female HR 0.48; 95\% CI 0.11-2.14 and male HR 2.79; 95\% CI 0.55-14.25). This result could be, in part, due to a preponderance of females in the sensitivity analyses versus the main cohort (77\% females in the sensitivity analysis versus $67 \%$ females in the main cohort).

\section{Discussion}

In this retrospective cohort study, we found that SCT was associated with an increased risk for CAD in male African American CKD patients. To our knowledge, this is the first study to look at cardiovascular outcomes in African American SCT patients with CKD.

Our findings suggest that sex differences in outcomes in SCD patients may also be seen in certain outcomes in SCT patients. Although SCD is more severe than SCT due to higher levels of hemoglobin S, they share similar physiological mechanisms - a cascade of inflammatory effects caused by the abnormal sickling of red blood cells in the microvasculature. In SCD, adult females are noted to have fewer pain crises and lower mortality rates compared to adult males [11-14]. Gladwin et al. [12] hypothesized that this could be related to estrogen-mediated effects and increased responsiveness to nitric oxide in females that ameliorate sickling, endothelial dysfunction, and inflammation. Other studies have shown that hemoglobin F (which, in significant amounts, inhibits sickling and reduces frequency of sickle cell crises in SCD) is higher in females compared to males, both in the general population and in SCD [24-26]. No studies that we are aware of have examined the effect of sex on SCT complications or inflammation markers. Nonetheless, literature describing cardiac arrest in individuals with SCT include predominantly male populations [27-29]. The data on
96

Am J Nephrol 2019;49:93-102 DOI: $10.1159 / 000496058$
Olaniran/Eneanya/Allegretti/Zhao/ Achebe/Thadhani 
Table 2. Baseline characteristics of cohort by sex

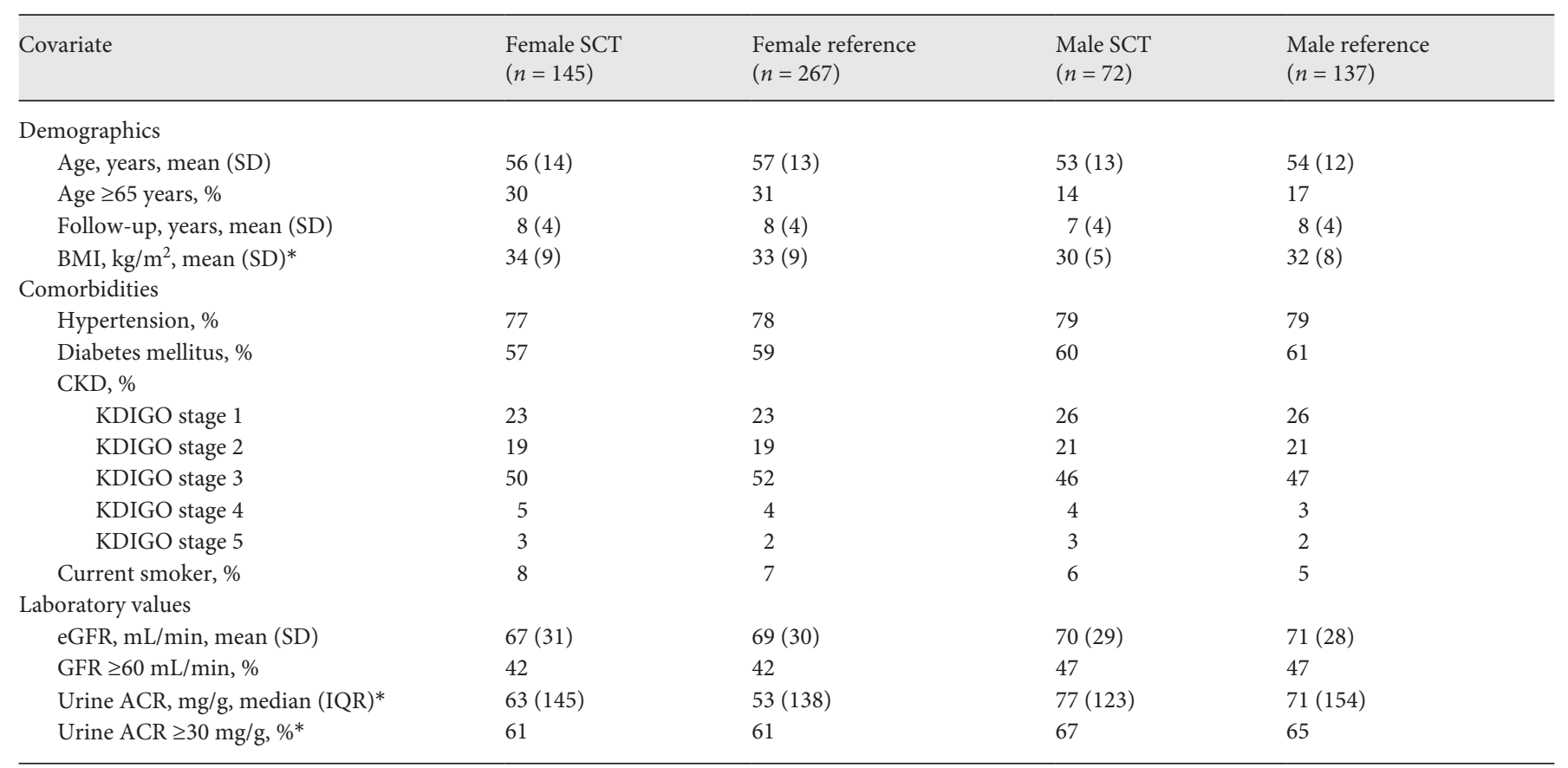

* Missing data were noted for BMI (152 patients) and urine albumin: creatinine ratio (120 patients).

Percentage values were calculated among whole cohort.

BMI, body mass index; CKD, chronic kidney disease; KDIGO, Kidney Disease Improving Global Outcomes; eGFR, estimated glomerular filtration rate; ACR, albumin creatinine ratio; SCT, sickle cell trait.

Table 3. Association of SCT and CKD with primary outcomes (all patients)

\begin{tabular}{|c|c|c|c|c|c|c|}
\hline & \multicolumn{6}{|c|}{ Outcomes (HR; 95\% CI) } \\
\hline & SCT & reference & SCT & reference & SCT & reference \\
\hline Events, $n(\%)$ & $20(43)$ & $19(78)$ & $11(24)$ & $16(33)$ & $10(22)$ & $7(28)$ \\
\hline Unadjusted & $1.13(0.78-1.64)$ & & $0.73(0.46-1.17)$ & & $1.51(0.87-2.64)$ & \\
\hline Adjusted & $1.16(0.79-1.68)$ & & $0.74(0.46-1.18)$ & & $1.52(0.87-2.67)$ & \\
\hline Hypertension & $0.93(0.54-1.59)$ & & $0.88(0.46-1.07)$ & & $0.47(0.23-0.95)$ & \\
\hline Diabetes mellitus & $1.49(0.99-2.23)$ & & $1.59(0.99-2.56)$ & & $1.17(0.64-2.16)$ & \\
\hline Baseline GFR & $1.00(0.99-1.01)$ & & $0.99(0.98-1.00)$ & & $0.97(0.95-0.98)$ & \\
\hline Interaction term: SCT + sex & $0.36(0.17-0.78)$ & $p<0.01$ & $2.06(0.72-5.89)$ & $p=0.18$ & $0.85(0.26-2.74)$ & $p=0.79$ \\
\hline
\end{tabular}

All outcomes were adjusted for age, sex, hypertension, diabetes mellitus, and baseline eGFR.

SCT, sickle cell trait; CKD, chronic kidney disease; CAD, coronary artery disease; eGFR, estimated glomerular filtration rate.

CAD in individuals with SCT are both conflicting and lacking in sub-analysis with regards to sex. Hyacinth et al. [30] performed a meta-analysis of 5 prospective cohorts investigating the association between SCT and CAD in 20,053 African Americans with varied renal function and found an increased risk for CAD (RR 1.42; 95\% CI 1.021.98). However, in one study in this meta-analysis, SCT patients in the Women's Health Initiative cohort had a lower risk for CAD (RR 1.05; 95\% CI 0.63-1.74) compared to the other cohorts [30]. A Kaiser Permanente 


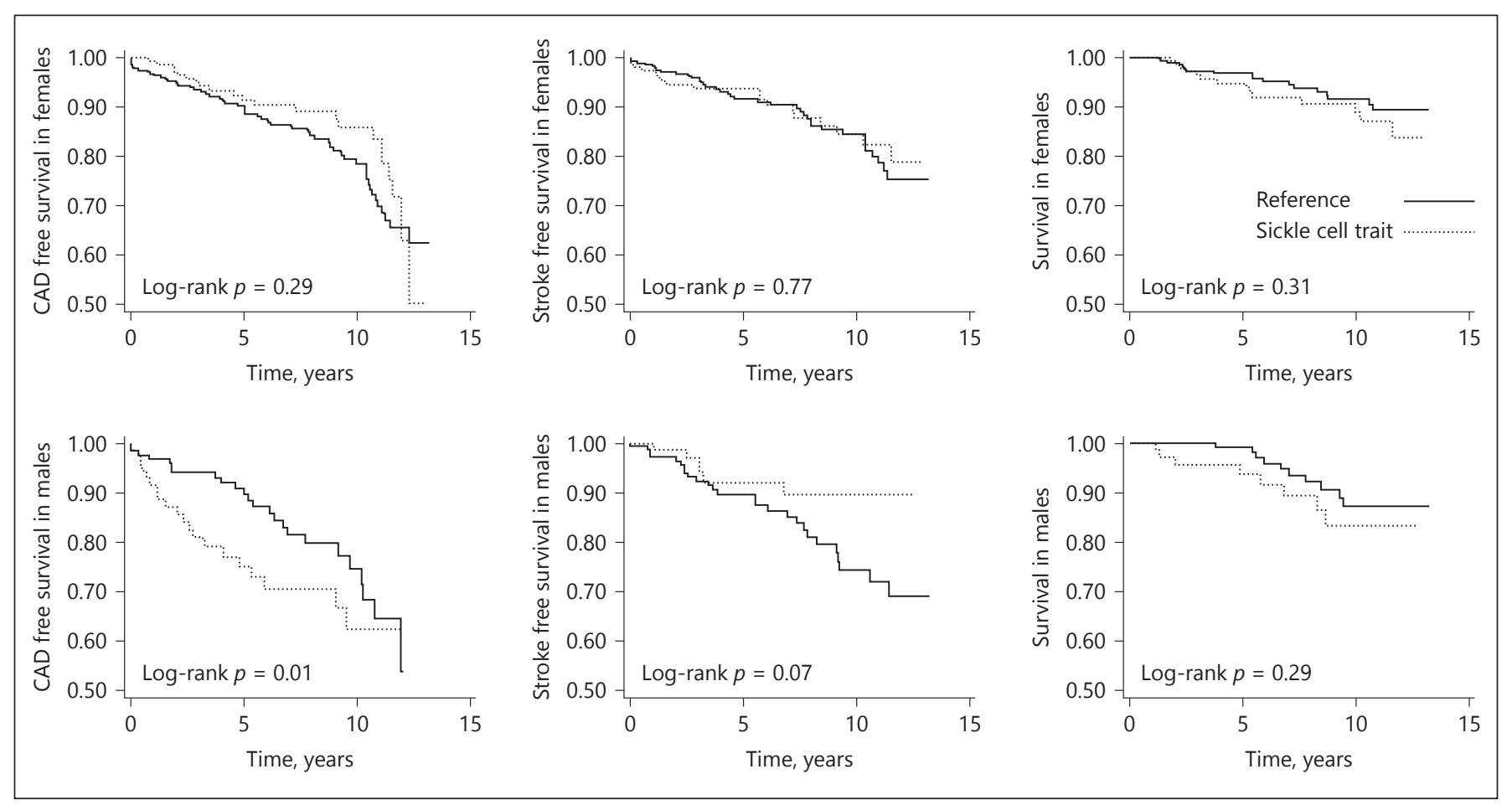

Fig. 2. Unadjusted association of SCT and CKD with outcomes by sex. CAD, coronary artery disease.

Table 4. Association of SCT and CKD with outcomes by sex

\begin{tabular}{|c|c|c|c|c|}
\hline & SCT & reference & SCT & reference \\
\hline Unadjusted CAD & $0.76(0.46-1.26)$ & - & $2.14(1.18-3.86)$ & - \\
\hline Adjusted CAD & $0.77(0.47-1.26)$ & - & $2.14(1.18-3.90)$ & - \\
\hline Stroke events, $n(\%)$ & $12(18)$ & $14(37)$ & $8(6)$ & $19(26)$ \\
\hline Mortality events, $n(\%)$ & $10(14)$ & $7(18)$ & $11(8)$ & $7(10)$ \\
\hline Unadjusted mortality & $1.43(0.71-2.87)$ & - & $1.64(0.65-4.16)$ & - \\
\hline Adjusted mortality & $1.39(0.69-2.81)$ & - & $1.61(0.63-4.13)$ & - \\
\hline
\end{tabular}

All outcomes were adjusted for age, hypertension, diabetes mellitus, and baseline eGFR.

SCT, sickle cell trait; CKD, chronic kidney disease; CAD, coronary artery disease.

study of 13,964 African Americans with varied renal function, which found no association between SCT and CAD, reported $77.2 \%$ of their population was female [7]. Neither the meta-analysis study nor the Kaiser Permanente study performed a stratified analysis of outcomes based on sex. More data are needed to better understand the role of sex in SCT outcomes.
Although modified by biological sex differences, the risk for CAD in SCT patients with CKD may be explained in part by inflammation and endothelial dysfunction due to sub-clinical sickling $[4,5]$. The renal medulla is well known to be an ideal environment for sickling due to vascular stasis, low oxygen partial pressures, and low $\mathrm{pH}$ [31]. Similar sub-clinical sickling may occur in other mi- 
Fig. 3. Adjusted association of SCT and CKD with outcomes by sex. All outcomes were adjusted for age, hypertension, diabetes mellitus and baseline eGFR. CAD, coronary artery disease $\mathrm{HR}$, hazard ratio; eGFR, estimated glomerular filtration rate.

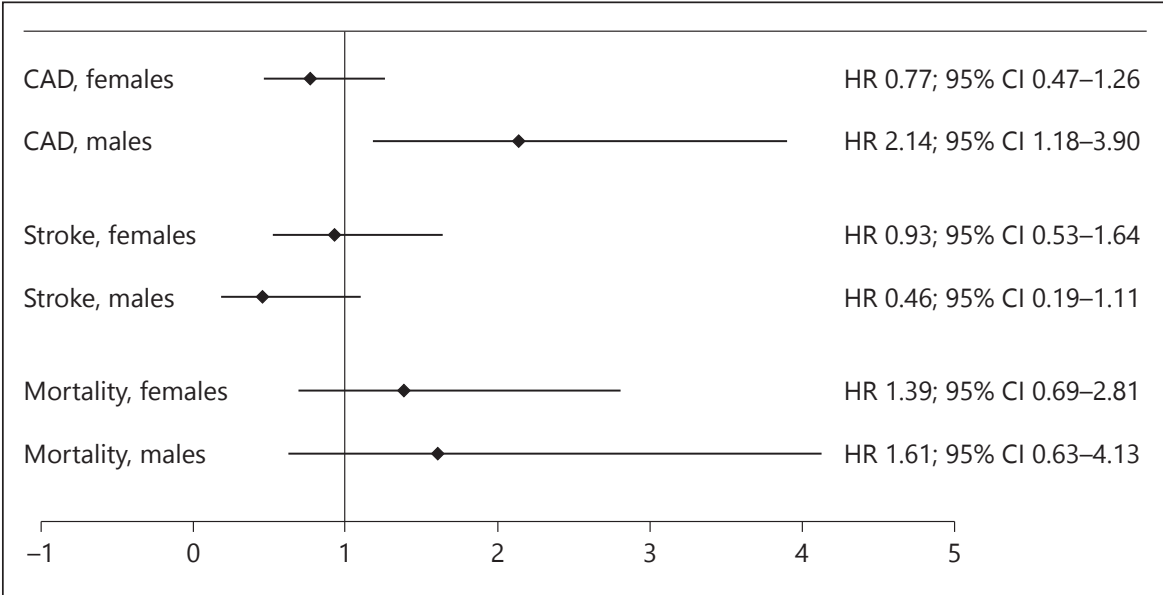

crovascular beds [32]. It has been proposed that low levels of inflammation caused by SCT can contribute to CAD in scenarios where a second hit - most commonly severe exertion - provokes the release of pro-inflammatory substances $[27,28]$. The concurrent presence of CKD, itself an independent risk factor for inflammation, endothelial dysfunction and CVD [33,34], may also offer a second hit and therefore exacerbate the risk for CAD in SCT. Prior studies examining the association between CAD and SCT have not addressed the superimposition of CKD $[7,30]$.

In terms of stroke, a study from the ARIC cohort observed an increased risk for stroke in SCT patients compared to African American patients without SCT (HR 1.4; 95\% CI 1.0-2.0) [35]. We found the opposite trend in our cohort, but this did not reach significance - perhaps due to a smaller number of events. Only one prior study looked at mortality in 119 SCT patients in Jamaica and found no significant differences with non-SCT patients [3]. This was not a CKD focused study.

Our study was limited by the fact that there were few outcome events. Other limitations included patients from a single geographic region, retrospective design of the study, and the use of ICD codes to ascertain exposure status which may limit generalizability. These data are best applied to those self-identifying as AA, and additional studies of other races would be needed to extrapolate our findings to those populations. We could not assess SCT exposure in the reference group which could bias the results towards the null. Finally, we were also unable to use hyperlipidemia status due to low sensitivity and specificity in our cohort. Nonetheless, rates of CAD and stroke were similar to previously described literature in the African American CKD population [36]. Despite these limitations, our results highlight important associations that merit fur-

Cardiovascular Outcomes in African

American SCT Kidney Disease the findings in this study have significant implications for our approach to screening and managing cardiovascular risk in African American CKD patients with SCT.

In conclusion, our study observed that SCT in African Americans with CKD is associated with an increased risk for CAD in males. This risk does not extend to African American females with SCT and CKD. These findings need to be confirmed in larger studies and further investigated to identify risk factors, best practices, and interventions to prevent adverse cardiovascular outcomes in African American SCT patients with CKD.

\section{Acknowledgments}

We would like to thank Dr. Sahir Kalim for his valuable contributions to manuscript preparation.

\section{Ethics Statement}

This study was approved by the Institutional Review Board at Partners Healthcare, Boston and the need for informed consent was waived.

\section{Disclosure Statement}

R.I.T. is a consultant to Fresenius Medical Care, North America.

\section{Funding Source}

K.O.O. is supported by the Ben J. Lipps Research Fellowship Award of the American Society of Nephrology. N.D.E. is supported by NIH grant K23DK114526-01. A.S.A is supported by ther investigation in larger prospective studies. We believe 
the American Heart Association Career Development Award 18CDA34110131. R.I.T. is supported by NIH grant R01DK094486-06.

\section{Author Contributions}

All authors were involved in study design, data analysis, and manuscript preparation.

\section{Appendix}

Sensitivity Analysis: Unadjusted outcomes based on patients with exposure status confirmed by hemoglobin electrophoresis $(n=61)$

Table A1. Association of SCT and CKD with primary outcomes (all patients with a hemoglobin electrophoresis)

\begin{tabular}{|c|c|c|c|c|c|c|}
\hline & \multicolumn{6}{|c|}{ Outcomes (HR; 95\% CI) } \\
\hline & $\begin{array}{l}\text { SCT } \\
(n=19)\end{array}$ & $\begin{array}{l}\text { reference } \\
(n=42)\end{array}$ & $\begin{array}{l}\text { SCT } \\
(n=19)\end{array}$ & $\begin{array}{l}\text { reference } \\
(n=42)\end{array}$ & $\begin{array}{l}\text { SCT } \\
(n=19)\end{array}$ & $\begin{array}{c}\text { reference } \\
(n=42)\end{array}$ \\
\hline Events, $n(\%)$ & $36(5)$ & $75(18)$ & $11(2)$ & $24(10)$ & $11(2)$ & $5(2)$ \\
\hline Unadjusted & $0.88(0.32-2.37)$ & - & $0.55(0.12-2.54)$ & - & $2.73(0.38-19.66)$ & - \\
\hline
\end{tabular}

SCT, sickle cell trait; CKD, chronic kidney disease; CAD, coronary artery disease.

Table A2. Association of SCT and CKD with outcomes by sex (all patients with a hemoglobin electrophoresis)

\begin{tabular}{|c|c|c|c|c|}
\hline & \multicolumn{2}{|c|}{ Female (HR; 95\% CI) } & \multicolumn{2}{|l|}{ Male (HR; 95\% CI) } \\
\hline Unadjusted CAD & $0.48(0.11-2.14)$ & - & $2.79(0.55-14.25)$ & - \\
\hline Stroke events, \% & 15 & 24 & 0 & 22 \\
\hline Unadjusted stroke & $0.80(0.17-3.78)$ & - & $* *$ & - \\
\hline
\end{tabular}

** Inadequate data for analysis.

SCT, sickle cell trait; CKD, chronic kidney disease; CAD, coronary artery disease.

Algorithms for Adjudicating Outcomes and Covariates Using ICD Codes

Coronary Artery Disease

The presence of at least 2 diagnosis codes during follow-up was required. We used the following codes: 410.x, 411.x, I20.0, I21.x, I24.x, I25.1x, I25.7x. These codes were evaluated on a subset of 696 African American CKD patients from the same database with CAD confirmed on chart review (using results of cardiac catheterizations, stress tests, and/or physician documentation of a myocardial infarction). Sensitivity: $81 \%$, Specificity: $81 \%$, negative predictive value (NPV): $93 \%$, positive predictive value (PPV): $57 \%$.
Stroke

The presence of at least 2 diagnosis codes during follow-up was required. We used the following codes: 362.30, 362.31, 362.32, 362.33, 362.34, 433.x, 434.x, 435.x, 436.x, 431.x, G45.x, H34.1x, I63.x, I65.x, I61.x. These codes were evaluated on a subset of 696 African American CKD patients from the same database with stroke confirmed on chart review (using brain imaging radiology reports and/or physician documentation of hemorrhagic or embolic stroke). Sensitivity: 87\%, Specificity: 88\%, NPV: 97\%, PPV: 59\%.

\section{Hypertension}

The presence of at least 15 diagnosis codes at different times during follow-up was required. We used the following codes: 997.91, 401.0, 401.1, 401.9, 402.00, 402.01, 402.10, 402.91, 402.90, 
403.01, 403.00, 404.01, 404.03, 404.91, 404.92, 404.93, 404.11, 404.13, 404.12, 404.10, 402.11, 403.11, 403.10, I10.x, I11.x, I12.x, I13.x, I15.x. These codes were evaluated on a subset of 696 African American CKD patients from the same database with hypertension confirmed on chart review (using multiple physician notes). Sensitivity: 80\%, Specificity: 76\%, NPV: 36\%, PPV: 96\%.

\section{Diabetes Mellitus}

The presence of at least 5 diagnosis codes at different times during follow-up was required. We used the following codes: 250.x, E10.x, E11.x, E12.x, E13.x, E14.x. These codes were evaluated on a subset of 696 African American CKD patients from the same database with diabetes mellitus confirmed on chart review (using multiple physician notes). Sensitivity: 98\%, Specificity: 82\%, NPV: $97 \%$, PPV: $86 \%$.

\section{End Stage Renal Disease}

The presence of at least 5 diagnosis codes during follow-up was required. We used the following codes: 585.6, N18.6. These codes were evaluated on a subset of 696 African American CKD patients from the same database with end stage renal disease confirmed on chart review (using multiple physician notes). Sensitivity: 96\%, Specificity: 92\%, NPV: 99\%, PPV: 69\%.

\section{Algorithms for Adjudicating Exposure Status Using ICD Codes}

\section{Sickle Cell Trait}

The presence of at least 1 diagnosis code at any time was required. We used the following codes: 282.5, D57.3.

\section{Sickle Cell Disease}

The presence of at least 1 diagnosis code at any time led to exclusion from this study. We used the following codes: 282.41, $282.42,282.60,282.61,282.62,282.63,282.64,282.68,282.69$, 289.52, 517.3, D57.00, D57.01, D57.02, D57.1, D57.20, D57.211, D57.212, D57.219, D57.40, D57.411, D57.412, D57.419, D57.80, D57.811, D57.812, D57.819.

\section{References}

1 Centers for Disease Control and Prevention. Data and Statistics | Sickle Cell Disease | NCBDDD | CDC [Internet]. Sickle Cell Disease Homepage. 2016 [cited 2017 Jun 8]. p. 1. Available from: https://www.cdc.gov/ncbddd/ sicklecell/data.html

2 United States Census Bureau US. Census Bureau QuickFacts: United States. https://www. census.gov/quickfacts/fact/table/US/ PST045216 (accessed 24 January 2018).

3 Ashcroft M, Desai P: Mortality and morbidity in Jamaican adults with sickle-cell trait and with normal haemoglobin followed up for twelve years. Lancet 1976;308:784-786.

4 Naik RP, Wilson JG, Ekunwe L, et al: Elevated D-dimer levels in African Americans with sickle cell trait. Blood 2016;127:2261-2263.

5 Amin C, Adam S, Mooberry MJ, et al: Coagulation activation in sickle cell trait: an exploratory study. Br J Haematol 2015;171:638646.

6 Carnethon MR, Pu J, Howard G, et al: Cardiovascular health in African Americans: a scientific statement from the American Heart Association. Circulation 2017;136:e393-e423.

7 Bucknor MD, Goo JS, Coppolino ML: The risk of potential thromboembolic, renal and cardiac complications of sickle cell trait. Hemoglobin 2014;38:28-32.

8 Naik RP, Derebail VK, Grams ME, et al: Association of sickle cell trait with chronic kidney disease and albuminuria in African Americans. JAMA 2014;312:2115-25.

9 Naik RP, Irvin MR, Judd S, et al: Sickle cell trait and the risk of ESRD in blacks. J Am Soc Nephrol 2017; 28:2180-2187.

10 Go AS, Chertow GM, Fan D, et al: Chronic kidney disease and the risks of death, cardio- vascular events, and hospitalization. $\mathrm{N}$ Engl J Med 2004;351:1296-1305.

11 Platt OS, Brambilla DJ, Rosse WF, et al: Mortality in sickle cell disease. Life expectancy and risk factors for early death. N Engl J Med 1994; 330:1639-1644

12 Gladwin MT, Schechter AN, Ognibene FP, et al: Divergent nitric oxide bioavailability in men and women with sickle cell disease. Circulation 2003;107:271-278.

13 Baum KF, Dunn DT, Maude GH, Serjeant GR: The painful crisis of homozygous sickle cell disease. a study of the risk factors. Arch Intern Med 1987;147:1231-1234.

14 Platt OS, Thorington BD, Brambilla DJ, et al: Pain in sickle cell disease. Rates and risk factors. N Engl J Med 1991;325:11-16.

15 Jolly SE, Burrows NR, Chen SC, et al: Racial and ethnic differences in mortality among individuals with chronic kidney disease: results from the Kidney Early Evaluation Program (KEEP). Clin J Am Soc Nephrol 2011;6:1858-1865.

16 Lash JP, Ricardo AC, Roy J, et al: Race/ethnicity and cardiovascular outcomes in adults with CKD: findings from the CRIC (Chronic Renal Insufficiency Cohort) and hispanic CRIC studies. Am J Kidney Dis 2016;68:545553.

17 Nalichowski R, Keogh D, Chueh HC, Murphy $\mathrm{SN}$ : Calculating the benefits of a research patient data repository. AMIA Annu Symp Proc 2006; 1044 .

18 Liao KP, Cai T, Gainer V, et al: Electronic medical records for discovery research in rheumatoid arthritis. Arthritis Care Res (Hoboken) 2010;62:1120-1127.

19 Nigwekar SU, Solid CA, Ankers E, et al Quantifying a rare disease in administrative data: the example of calciphylaxis. J Gen Intern Med 2014;29:S724-S731.

20 Kidney Disease: Improving Global Outcomes (KDIGO) CKD Work Group. KDIGO 2012 Clinical Practice Guideline for the Evaluation and Management of Chronic Kidney Disease KDIGO 2012 Clinical Practice Guideline for the Evaluation and Management of Chronic Kidney Disease. Kidney Int 2013;3:1-150.

21 Levey AS, Bosch JP, Lewis JB, et al: A more accurate method to estimate glomerular filtration rate from serum creatinine: a new prediction equation. Modification of Diet in Renal Disease Study Group. Ann Intern Med 1999; 130:461-470.

22 Inker LA, Schmid CH, Tighiouart H, et al: Estimating glomerular filtration rate from serum creatinine and cystatin C. N Engl J Med 2012;367:20-29.

23 Kellum JA, Lameire N, Aspelin P, et al: KDIGO clinical practice guideline for acute kidney injury. Kidney Int Suppl 2012;2:1-138.

24 Chang YC, Smith KD, Moore RD, et al: An analysis of fetal hemoglobin variation in sickle cell disease: the relative contributions of the $\mathrm{X}$-linked factor, beta-globin haplotypes, alpha-globin gene number, gender, and age. Blood 1995;85:1111-1117.

25 Chang YP, Maier-Redelsperger M, Smith $\mathrm{KD}$, et al: The relative importance of the $\mathrm{X}$ linked FCP locus and beta-globin haplotypes in determining haemoglobin F levels: a study of SS patients homozygous for beta S haplotypes. Br J Haematol 1997;96:806-814.

26 Akinsheye I, Alsultan A, Solovieff N, et al: Fetal hemoglobin in sickle cell anemia. Blood 2011;118:19-27. 
27 Murray SW, Cooper RM, Mills JD, Palmer ND: Evading the fate of Pheidippides: acute coronary thrombosis in a young marathon runner with minimal atherosclerosis but sickle cell trait. Cardiovasc Diagn Ther 2015;5:330-334

28 Dahhan A, Wang X, Mandawat M, Robinson VJ: Myocardial infarction after physical exertion in a healthy young patient with coronary artery ectasia and sickle cell trait. Int J Cardiol 2015;190:111-113.

29 Harris KM, Haas TS, Eichner ER, Maron BJ: Sickle cell trait associated with sudden death in competitive athletes. Am J Cardiol 2012; 110:1185-1188.
30 Hyacinth HI, Cara CL, Seals SR, et al: Association of sickle cell trait with risk of coronary heart disease in African Americans. Blood 2016;128:11.

31 Nath KA, Hebbel RP: Sickle cell disease: renal manifestations and mechanisms. Nat Rev Nephrol 2015;11:161-171.

32 Podduturi V, Guileyardo JM: Sickle cell trait as a contributory cause of death in natural disease. J Forensic Sci 2015;60:807-811.

33 Knight EL, Rimm EB, Pai JK, et al: Kidney dysfunction, inflammation, and coronary events: a prospective study. J Am Soc Nephrol 2004;15:1897-1903.
34 Debella YT, Giduma HD, Light RP, Agarwal $\mathrm{R}$ : Chronic kidney disease as a coronary disease equivalent-a comparison with diabetes over a decade. Clin J Am Soc Nephrol 2011;6: 1385-1392.

35 Caughey MC, Loehr LR, Key NS, et al: Sickle cell trait and incident ischemic stroke in the atherosclerosis risk in communities study. Stroke 2014;45:2863-2867.

36 Bansal N, Katz R, Robinson-Cohen C, et al: Absolute rates of heart failure, coronary heart disease, and stroke in chronic kidney disease: an analysis of 3 community-based cohort studies. JAMA Cardiol 2017;2:314-318. 